\title{
Universiteit
}

Leiden

The Netherlands

\section{Quantification of bone involvement in Gaucher disease: MR imaging bone marrow burden score as an alternative to dixon quantitative chemical shift MR imaging - Initial experience \\ Maas, M.; Kuijk, C. van; Stoker, J.; Hollak, C.E.M.; Akkerman, E.M.; Aerts, J.F.M.G.; Heeten, G.J. den}

\section{Citation}

Maas, M., Kuijk, C. van, Stoker, J., Hollak, C. E. M., Akkerman, E. M., Aerts, J. F. M. G., \& Heeten, G. J. den. (2003). Quantification of bone involvement in Gaucher disease: MR imaging bone marrow burden score as an alternative to dixon quantitative chemical shift MR imaging - Initial experience. Radiology, 229(2), 554-561. doi:10.1148/radiol.2292020296

Version: $\quad$ Publisher's Version

License: $\quad$ Leiden University Non-exclusive license

Downloaded from: https://hdl.handle.net/1887/81194

Note: To cite this publication please use the final published version (if applicable). 
Mario Maas, MD, PhD Cornelis van Kuijk, MD, PhD Jaap Stoker, MD, PhD Carla E. M. Hollak, MD, PhD Erik M. Akkerman, PhD Johannes F. M. G. Aerts, PhD Gerard J. den Heeten, MD, $\mathrm{PhD}$

Index terms:

Bone marrow, diseases, 33.671, 45.671

Bone marrow, MR, 33.1214, 45.1214

Gaucher disease, 33.671, 45.671

Magnetic resonance (MR), chemical shift

Published online before print 10.1148/radiol.2292020296

Radiology 2003; 229:554-561

Abbreviations:

$\mathrm{BMB}=$ bone marrow burden

$\mathrm{QCSI}=$ quantitative chemical shift imaging

${ }^{1}$ From the Departments of Radiology (M.M., C.v.K., J.S., E.M.A., G.J.d.H.), Hematology (C.E.M.H.), and Biochemistry (J.F.M.G.A.), Academic Medical Center, Meibergdreef 9, Suite G1-231, 1105 AZ Amsterdam, the Netherlands. Received April 17, 2002; revision requested July 8; final revision received March 13, 2003; accepted April 7. Address correspondence to M.M. (e-mail: m.maas@amc uva.nl).
Author contributions:

Guarantor of integrity of entire study, M.M.; study concepts, all authors; study design, M.M., C.v.K., J.S., C.E.M.H., E.M.A., G.J.d.H.; literature research, M.M., C.E.M.H., E.M.A.; clinical studies, M.M., C.v.K., J.S., C.E.M.H.; data acquisition, M.M., C.v.K., J.S.; data analysis/ interpretation, all authors; statistical analysis, M.M., C.v.K., C.E.M.H., E.M.A., G.J.d.H.; manuscript editing, M.M., C.v.K., J.S., J.F.M.G.A., G.J.d.H.; manuscript preparation and definition of intellectual content, revision/review, and final version approval, all authors

o RSNA, 2003

\section{Quantification of Bone} Involvement in Gaucher Disease: MR Imaging Bone Marrow Burden Score as an Alternative to Dixon Quanti-
tative Chemical Shift MR
Imaging-Initial Experience

PURPOSE: To develop a semiquantitative magnetic resonance (MR) imaging bone marrow burden (BMB) score with inclusion of both axial and peripheral bone marrow in Gaucher disease as an alternative to MR imaging with the Dixon quantitative chemical shift imaging (QCSI) technique.

MATERIALS AND METHODS: Two experienced musculoskeletal radiologists with no experience in evaluating Gaucher disease blindly analyzed MR images of lumbar spines and femora. Interobserver and intraobserver variability were tested. In addition, the BMB score was determined as a parameter to evaluate bone marrow response to enzyme supplementation therapy. Finally, the BMB score was compared with fat fraction measurements obtained with Dixon QCSI. Differences between groups were analyzed by using the nonparametric Mann-Whitney test. A $P$ value of less than .05 was considered to represent significance. Correlation was calculated by using two-tailed nonparametric rank correlation (Spearman $\rho$ ).

RESULTS: In 30 patients (mean age, 39.3 years; age range, 12-71 years) the mean fat fraction was 0.20 (range, $0.08-0.40$ ). The BMB score range was 3-13 points. A significant correlation was found between the two observers when using $B M B$ $(\rho=0.91, P<.001)$. The intraobserver variation showed a significant correlation $(\rho=0.99, P<.001)$. There was a significant correlation between BMB and QCSI $(\rho=-0.78, P<.001)$. Although BMB was less sensitive than Dixon QCSI, it showed enough sensitivity to allow detection of bone marrow response to enzyme supplementation therapy.

CONCLUSION: BMB is a reproducible semiquantitative scoring system that is easy to use. It combines MR imaging of both axial and peripheral bone marrow and shows a significant correlation with QCSI.

○ RSNA, 2003

Gaucher disease is the most prevalent lysosomal storage disorder in humans, and it leads to the storage of glycocerebrosidase-loaded macrophages in the bone marrow, liver, and spleen (1-3). The clinical features of type 1 disease encompass hepatosplenomegaly, cytopenia, and bone involvement (3). Bone disease is one of the most debilitating features of type 1 Gaucher disease and consists of atypical bone pain, osteonecrosis, pathologic fractures, and bone crises $(2,4)$. Enzyme supplementation therapy for the treatment of Gaucher disease has become available during the past decade (5-7). Since this is a very expensive therapy, adequate monitoring of response to treatment is important in order to facilitate dose adjustments.

Magnetic resonance (MR) imaging is the modality of choice to depict bone marrow 

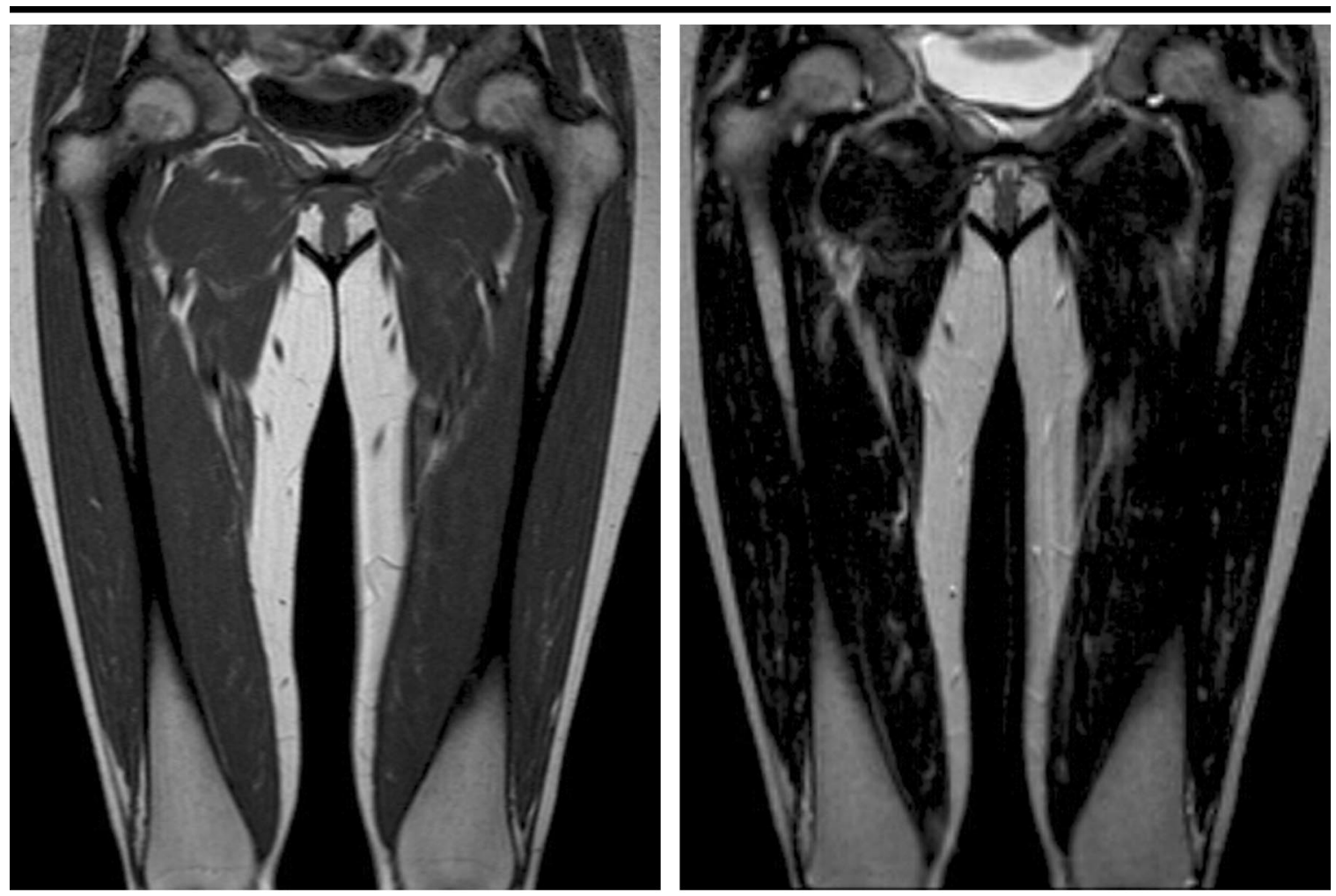

a.

b.

Figure 1. Coronal (a) T1- and (b) T2-weighted MR images of femora. There is only a slight decrease in signal intensity in the femoral metaphysis and diaphysis. The epiphysis shows no involvement.

abnormalities (8-14). It is also used to describe bone marrow invasion in a (semi) quantitative way. From the first MR imaging examination of a patient, the degree of bone marrow invasion is quantified, and an indication for enzyme supplementation therapy is considered. To quantitatively analyze bone marrow involvement and response to therapy, Dixon quantitative chemical shift imaging (QCSI) of the vertebral (axial) bone marrow is performed $(15,16)$. Dixon QCSI is a modification of the Dixon technique (17), and it quantifies the fat content in bone marrow by making use of the difference in the resonant frequencies between fat and water in bone marrow $(3.3 \mathrm{ppm})$. The amount of fat in the bone marrow is represented in a fat fraction $F_{f}$, which is decreased in Gaucher disease (15,17-21). The results of a recent study established a high reproducibility of this technique (22). Studies in which Dixon QCSI has been used have focused on vertebral bone marrow because it contains primarily red marrow, which is very susceptible to disease that arises from hematologic malignancies $(10,23-25)$. Furthermore, the axial marrow is the first site to demonstrate bone marrow infiltration in Gaucher disease $(16,21)$. There- fore, measuring the axial marrow may provide the most exact status of bone marrow invasion.

Although MR imaging is widely available, Dixon QCSI is not included in the standard packages of sequences on MR imagers and cannot be used worldwide. Therefore, several visual scoring systems have been used and described in the literature $(1,26-28)$. As a result of these visual scoring systems, a semiquantitative approach to bone marrow invasion has been established. These bone marrow scoring systems are restricted to evaluation of the peripheral bone marrow of the lower extremities. However, to our knowledge, none of the published semiquantitative scoring systems have evaluated axial bone marrow or have been compared with Dixon QCSI data.

In our opinion, an optimal scoring system should include evaluation of the axial skeleton. Furthermore, there should be good interobserver and intraobserver variability, and the scoring system should be reproducible and sensitive for response to therapy. To our knowledge, no semiquantitative bone marrow burden (BMB) score that includes the axial bone marrow has so far been reported.

The purpose of our study was to de-

\begin{tabular}{|c|c|c|}
\hline \multicolumn{3}{|c|}{ A: MR Imaging Signal Intensity } \\
\hline \multicolumn{3}{|l|}{ Relaxation } \\
\hline Time & Signal Intensity* & BMB Score \\
\hline T2 & Hyperintense & 2 \\
\hline T2 & Slightly hyperintense & 1 \\
\hline T2 & Isointense & 0 \\
\hline $\mathrm{T} 2$ & Slightly hypointense & 1 \\
\hline T2 & Hypointense & 2 \\
\hline $\mathrm{T} 2$ & Mixed type & 3 \\
\hline T1 & $\begin{array}{l}\text { Slightly hyperintense } \\
\text { or isointense }\end{array}$ & 0 \\
\hline T1 & Slightly hypointense & 1 \\
\hline T1 & Hypointense & 2 \\
\hline
\end{tabular}

Note.-A higher BMB score signifies more severe bone marrow involvement.

* Determined in relation to signal intensity of subcutaneous fat.

B: Sites of Involvement

Bone Site Involved BMB Score

Diaphysis

Proximal epiphysis/apophysis Distal epiphysis 3

Note.-A higher BMB score signifies more severe bone marrow involvement.

velop a semiquantitative $\mathrm{MR}$ imaging $\mathrm{BMB}$ score including both axial and 

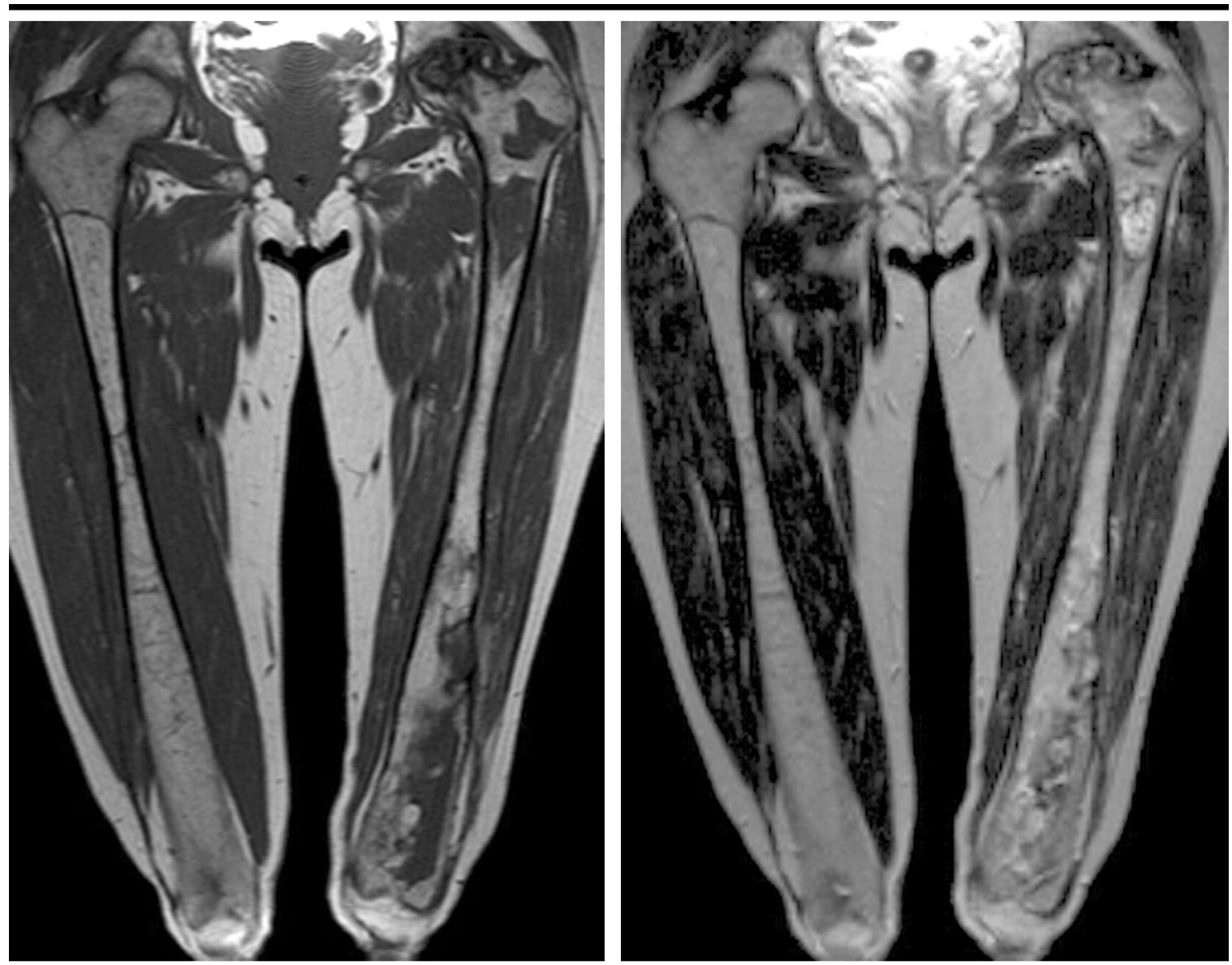

a.

b.

Figure 2. Coronal (a) T1- and (b) T2-weighted MR images of the femora in another patient. The left femur has low signal intensity on the T1-weighted image (a) and is an example of a mixed pattern, which is particularly present on the T2-weighted image (b).

peripheral bone marrow that could be used as an alternative to the Dixon QCSI technique in the evaluation of Gaucher disease.

\section{MATERIALS AND METHODS}

\section{Subjects}

Our hospital serves as a national referral center for Gaucher disease (6). Between 1993 and 1999, all untreated patients with type 1 Gaucher disease who were referred to undergo evaluation of eligibility for enzyme treatment were consecutively included in this study. The measurement of glucocerebrosidase activity in leukocytes and genotyping were used to confirm the diagnosis of Gaucher disease in all patients (29-31). All patients underwent Dixon QCSI of the lumbar spine as part of a complete diagnostic work-up that consisted of conventional T1- and T2-weighted turbo spin-echo MR imaging sequences of the lumbar spine and femora. This study was approved by our institutional review board, and informed consent was obtained.

\section{Theoretical Base of BMB Score}

In this study an alternative BMB scoring system was introduced; it is a combination of scoring systems of the peripheral skeleton already described and the axial bone marrow. In the literature, the semiquantitative scoring is based on two features: signal intensity changes and sites of involvement in the peripheral skeleton $(11,15,21,26,28)$. In Gaucher disease, the predominant MR imaging characteristic of infiltrated bone marrow is low signal intensity on T1-, T2-, and $\mathrm{T} 2{ }^{*}$ weighted images. Occasionally one may find an increase in signal intensity on T2-weighted images; this is thought to reflect active bone marrow disease such as osteonecrosis or infarction $(11,28)$. Furthermore, it has been shown that Gaucher disease spreads in bone in a reproducible manner, starting in the metadiaphysis and finally invading the epiphysis and apophysis $(15,21)$. The introduced BMB score incorporates both the visual interpretation of signal intensity and the geographic location of the disease on conventional MR images of the spine and femur. The chosen points given to the various parameters are arbitrary.

Lower extremities.- The signal intensity of bone marrow on T1- and T2-weighted images was graded in comparison with the signal intensity of subcutaneous fat by using a classification modified slightly from one used in earlier studies (Table 1) $(26,27)$. The levels of signal intensity were scored as hyperintense, slightly hyperintense, isointense, slightly hypointense, and hypointense. Gaucher disease is expected to demonstrate hypointense signal intensity changes $(9-11,21)$. In severe cases, a mixed pattern of signal intensity is seen in the extremities with areas of high and low signal intensity, 
TABLE 2

\section{Evaluation of $B M B$ in the Lumbar Spine}

A: MR Imaging Signal Intensity

\begin{tabular}{clc}
\hline $\begin{array}{c}\text { Relaxation } \\
\text { Time }\end{array}$ & \multicolumn{1}{c}{ Signal Intensity* } & BMB Score \\
\hline T2 & Hyperintense & 2 \\
T2 & Slightly hyperintense & 1 \\
T2 & Isointense & 0 \\
T2 & Slightly hypointense & 1 \\
T2 & Hypointense & 2 \\
T1 & Slightly hyperintense & 0 \\
T1 & Isointense & 1 \\
T1 & Slightly hypointense & 2 \\
T1 & Hypointense & 3 \\
\hline
\end{tabular}

Note.-A higher BMB score signifies more severe bone marrow involvement.

* Determined in relation to signal intensity of nondiseased intervertebral disk.

\section{B: Infiltration Pattern}

\begin{tabular}{lc}
\hline \multicolumn{1}{c}{ Pattern } & BMB Score \\
\hline Patchy & 1 \\
Diffuse & 2 \\
$\begin{array}{l}\text { Absence of fat in basivertebral } \\
\text { vein region }\end{array}$ & 1 \\
\hline
\end{tabular}

Note.-A higher BMB score signifies more severe bone marrow involvement.

especially on T2-weighted images. The areas of low signal intensity are thought to be due to Gaucher cell infiltration, whereas the areas of high signal intensity are thought to reflect acute complications such as infarction or bone crisis $(11,21)$. In our study, there were three sites of involvement in the femora: proximal epiphysis and apophysis, (meta-)diaphysis, and distal epiphysis. This was a modification of the sites of involvement score $(15,21)$. Examples of mild and severe disease are shown in Figures 1 and 2.

Lumbar spine. - The T1-weighted images were evaluated by using a nondiseased intervertebral disk as a reference for isointensity (Table 2) (13). The bone marrow signal intensity on T2-weighted images was evaluated by using the presacral fat as a normal reference for isointensity. Gaucher disease was expected to show low signal intensity on T1- and T2weighted images $(9-11,21)$. In addition, the infiltration pattern (patchy or diffuse) of the disease within the vertebral bodies was graded. When MR imaging is used in the evaluation of hematologic malignancies, a distinction is made between bone marrow infiltration patterns in the lumbar spine $(13,28)$. For instance, in multiple myeloma an association between a diffuse infiltration pattern and more advanced disease is hypothesized
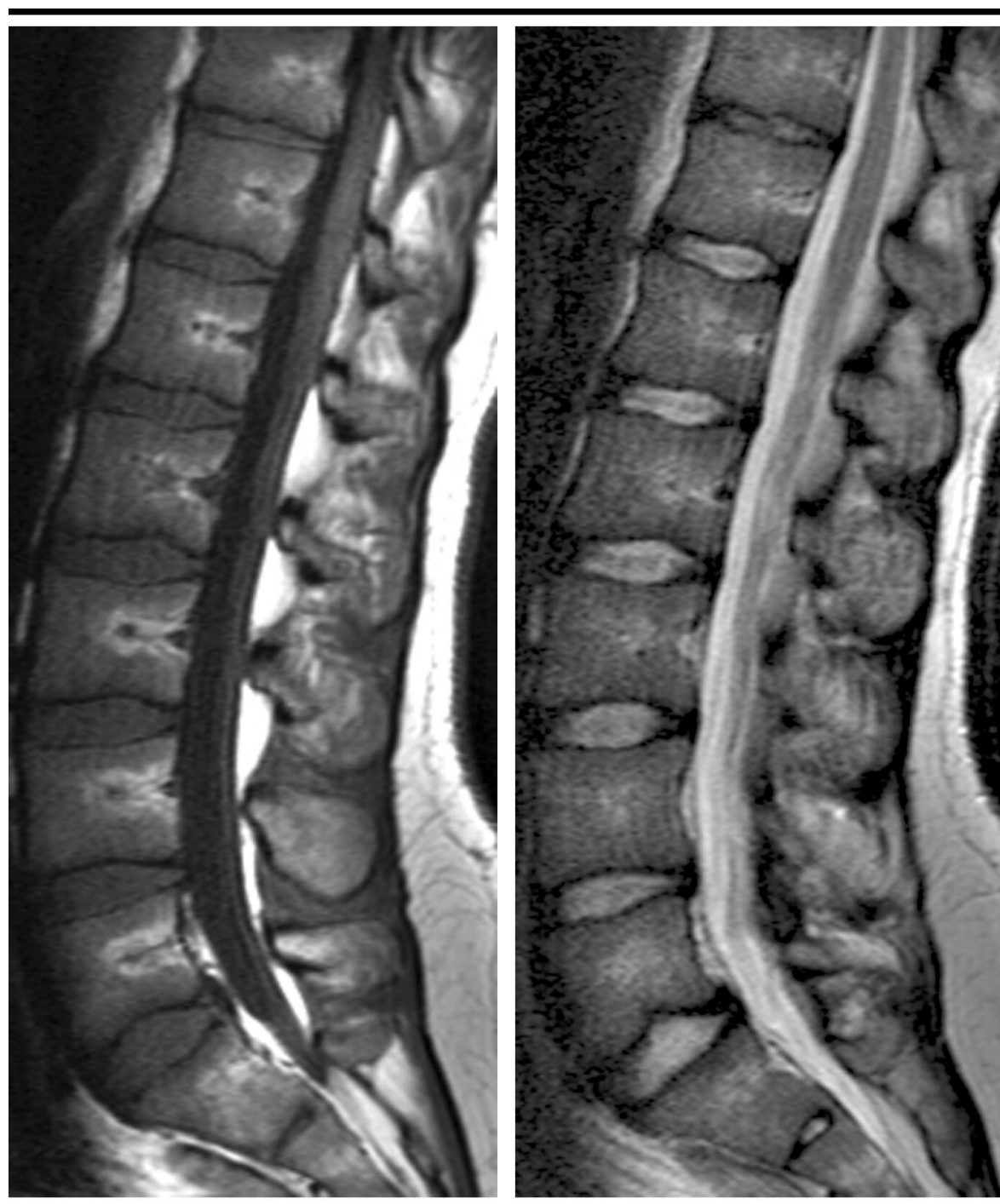

a.

b.

Figure 3. Sagittal (a) T1- and (b) T2-weighted MR images of the lumbar spine obtained in the same patient as in Figure 1. The lumbar spine shows more infiltration than the femora. There is marked low signal intensity on both T1- and T2-weighted images. The preservation of the fat surrounding the basivertebral vein is clearly appreciable.

$(10,12,32)$. This pattern can also be recognized in Gaucher disease. The patchy infiltration pattern seen in Gaucher disease consists of localized areas of abnormal bone marrow (low signal intensity on T1- and T2-weighted images) on a background of normal bone marrow (high signal intensity on T1- and T2-weighted images). With the diffuse pattern, the bone marrow is completely replaced; on T1-weighted images, bone marrow is hypointense or isointense to the intervertebral disk, and on T2-weighted images, a variable decrease in signal intensity of the abnormal marrow is seen. The diffuse pattern is considered to reflect a more advanced stage of the disease. Furthermore, we noted the absence of fat in the basivertebral vein region in a binominal fashion (present or absent). Disappearance of the fat surrounding the basivertebral vein at the site where it enters the vertebral body dorsally has been described as the first sign of bone marrow invasion in malignancy (33). Since disappearance of fat at this site had been observed in our group of patients with Gaucher disease, it was included as a parameter in the BMB scoring system. Examples of mild and severe disease are shown in Figures 3 and 4.

\section{BMB Evaluation}

Two musculoskeletal radiologists (J.S., C.v.K.), who were experienced in the analysis of MR images but were not expe- 


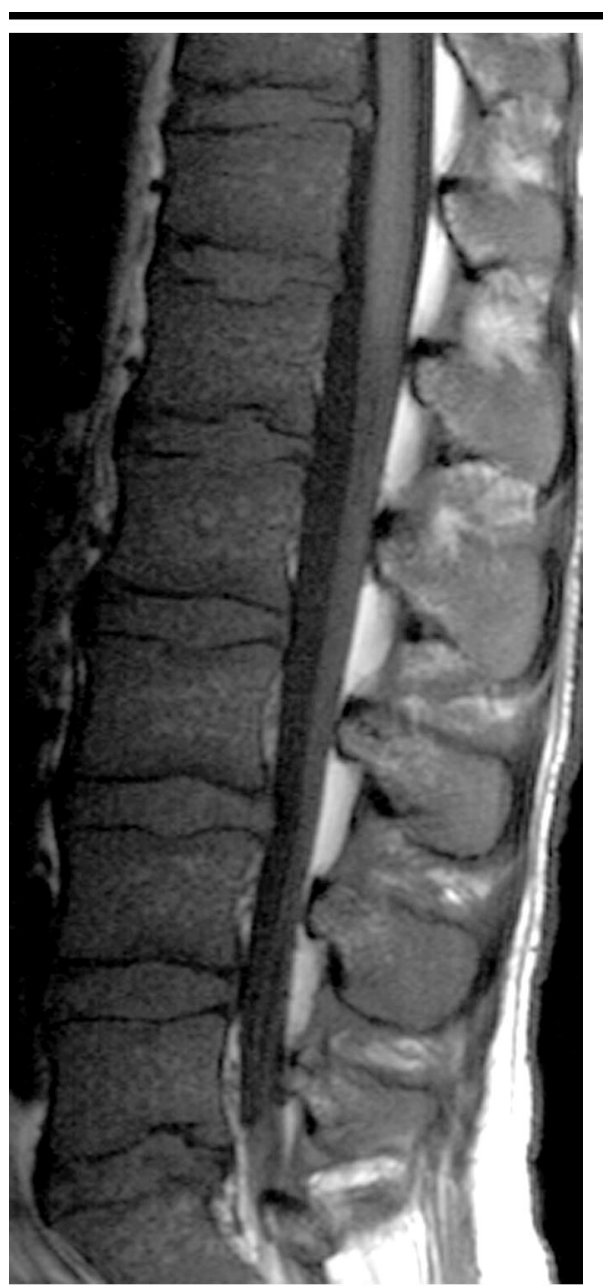

a.

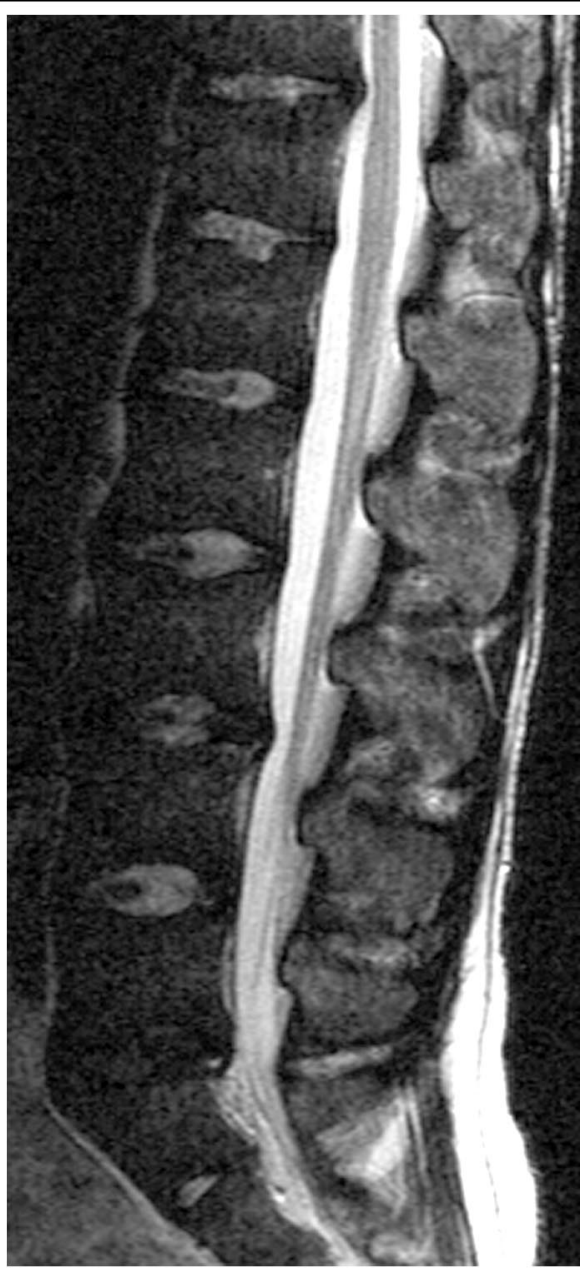

b.

Figure 4. Sagittal (a) T1- and (b) T2-weighted MR images of the lumbar spine in another patient. There is diffuse infiltration of the axial bone marrow seen on both $\mathbf{a}$ and $\mathbf{b}$, reflecting severe involvement.

rienced in the evaluation of Gaucher disease, separately reviewed the conventional MR images of both the femora and the lumbar spine retrospectively from hardcopy images. Both observers were blinded to patient characteristics and the examination date. In this way the interobserver variability was tested. To analyze the intraobserver variability, a small number of cases $(n=10)$ were chosen at random, and one observer (J.S.) evaluated them twice within an interval of 12 months. Both the lumbar spine and femora scores were added up, which led to a total score with a maximum of 16 (femora, eight; lumbar spine, eight). The final score was an aggregated score based on worst-case appearance. A higher score represented more severe bone marrow involvement (Tables 1, 2).

To evaluate the BMB score as a follow-up parameter, one observer (J.S.) evaluated the MR images of patients who were treated for 40 months or longer. These follow-up MR images were mixed with the baseline MR images to make the observer unaware of the time when they were obtained.

For our follow-up analysis, we defined a good response to therapy as an increase of at least three times the precision of the measurement (34). Since the precision of QCSI is measured at 3 points (22), we defined $2.8 \times 3$ points, which would make 9 points of increase in fat fraction a good response within 95\% confidence limits (34). The precision of BMB evaluation is discussed in the Results section. To analyze the separate contributions of the axial and peripheral bone marrow evaluations, we subdivided the BMB scores into the $\mathrm{BMB}$ lumbar spine and $\mathrm{BMB}$ femur scores.

\begin{tabular}{|c|c|c|c|c|}
\hline $\begin{array}{c}\text { Patient } \\
\text { No. }\end{array}$ & Sex & Age (y) & $\begin{array}{c}\text { Fat } \\
\text { Fraction }\end{array}$ & $\begin{array}{l}\text { BMB } \\
\text { Score }\end{array}$ \\
\hline 1 & $\mathrm{~F}$ & 30 & 0.13 & 13 \\
\hline 2 & M & 37 & 0.11 & 12 \\
\hline 3 & $\mathrm{M}$ & 57 & 0.34 & 12 \\
\hline 4 & $\mathrm{~F}$ & 39 & 0.11 & 13 \\
\hline 5 & M & 29 & 0.10 & 13 \\
\hline 6 & $\mathrm{~F}$ & 71 & 0.19 & 8 \\
\hline 7 & $M$ & 65 & 0.12 & 10 \\
\hline 8 & $\mathrm{~F}$ & 53 & 0.22 & 9 \\
\hline 9 & $\mathrm{M}$ & 38 & 0.10 & 9 \\
\hline 10 & $M$ & 53 & 0.23 & 10 \\
\hline 11 & $\mathrm{M}$ & 43 & 0.29 & 4 \\
\hline 12 & $\mathrm{M}$ & 48 & 0.40 & 4 \\
\hline 13 & $M$ & 52 & 0.18 & 9 \\
\hline 14 & $\mathrm{~F}$ & 42 & 0.22 & 7 \\
\hline 15 & $\mathrm{M}$ & 33 & 0.08 & 11 \\
\hline 16 & $\mathrm{~F}$ & 36 & 0.08 & 13 \\
\hline 17 & M & 44 & 0.15 & 12 \\
\hline 18 & $\mathrm{~F}$ & 45 & 0.18 & 9 \\
\hline 19 & $\mathrm{M}$ & 47 & 0.25 & 6 \\
\hline 20 & $\mathrm{~F}$ & 55 & 0.32 & 5 \\
\hline 21 & $M$ & 19 & 0.24 & 3 \\
\hline 22 & $\mathrm{~F}$ & 39 & 0.17 & 10 \\
\hline 23 & $\mathrm{~F}$ & 43 & 0.32 & 7 \\
\hline 24 & $\mathrm{~F}$ & 44 & 0.16 & 12 \\
\hline 25 & $\mathrm{~F}$ & 35 & 0.33 & 8 \\
\hline 26 & $M$ & 44 & 0.24 & 9 \\
\hline 27 & $\mathrm{~F}$ & 31 & 0.14 & 12 \\
\hline 28 & $M$ & 51 & 0.18 & 8 \\
\hline 29 & $M$ & 36 & 0.23 & 7 \\
\hline 30 & $\mathrm{~F}$ & 12 & 0.17 & 7 \\
\hline
\end{tabular}

\section{Dixon QCSI}

Dixon QCSI is a highly reproducible noninvasive technique that is based on the phase-contrast technique described by Dixon, in which the MR imaging signal intensity of bone marrow is separated into the individual contributions of fat signal and water signal. In this manner, it is possible to determine fat fractions $(17,19,22,25,35)$. To obtain fat fractions in the lumbar vertebrae L3 through L5, determination of regions of interest were manually drawn by one of the authors (E.M.A.) by following a protocol described previously, with an average size of 254 pixels (range, 153-343 pixels) $(22,35)$. Fat fraction was given as a percentage of the total volume of bone marrow. As such the fat fraction theoretically ranged from 0 (no fat) to 1 (only fat). In a group of 16 healthy volunteers, the mean fat fraction was measured to be 0.37 (SD, \pm 0.08$)(22)$.

\section{Statistical Analysis}

Data were analyzed by using SPSS 10-0 software (SPSS, Chicago, Ill). Differences between groups were analyzed by using the nonparametric Mann-Whitney test. 


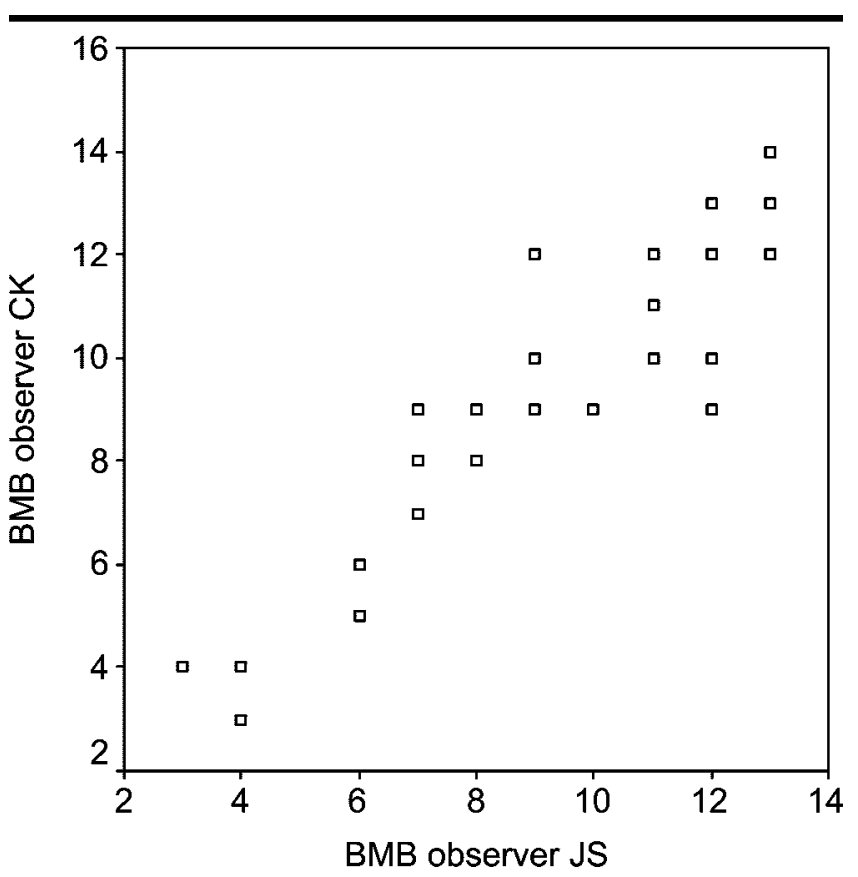

Figure 5. Graph shows correlation in BMB score between two observers $(\rho=0.91, P<.001)$.

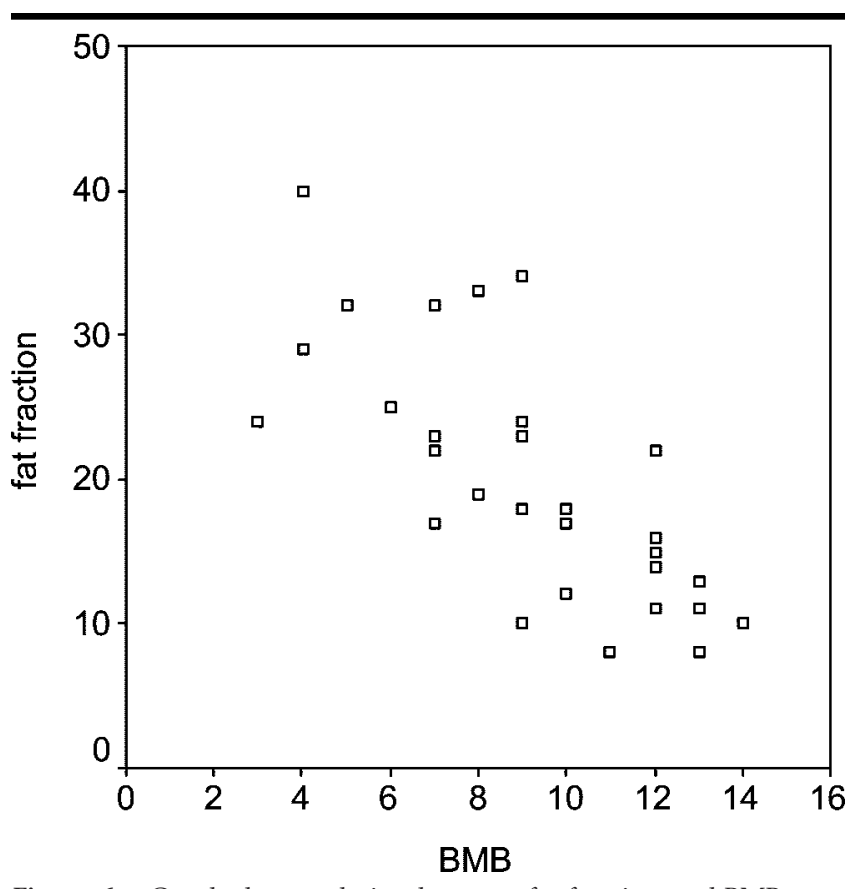

Figure 6. Graph shows relation between fat fraction and BMB scoring in untreated patients $(\rho=-0.78, P<.001)$.
A $P$ value of less than .05 was considered to represent significance. Correlation was calculated by using two-tailed nonparametric rank correlation (Spearman $\rho$ ).

\section{RESULTS}

Findings from 30 patients were analyzed (16 male patients, 14 female patients), and the mean patient age was 39.3 years (age range, 12-71 years). There was no significant difference in age distribution between the sexes (Mann-Whitney test). No patients were excluded or unable to finish the examination. The mean fat fraction in this population was 0.20 (range, $0.08-0.40)$. The BMB score range was 3-13 points. The patient characteristics at baseline examination are listed in Table 3 .

On the basis of the outcome of the BMB score, there was a significant correlation between the two observers ( $\rho=$ $0.91, P<.001)$ (Fig 5). There was also a significant correlation between fat fractions and BMB scores $(\rho=-0.78, P<$ .001) (Fig 6). When the MR images were analyzed twice by the same observer (J.S.), a significant correlation was found $(\rho=0.99, P<.001)$.

For longitudinal follow-up, the measurements obtained in 12 patients were analyzed for fat fraction and BMB score, with a follow-up interval of 40 months
(Table 4). The mean change in fat fraction was an increase of 0.17 (range, $0.08-$ 0.29). The mean change in $\mathrm{BMB}$ score was a decrease of 3 points (range, -9 to 2 points). According to our preset criteria, when fat fraction was considered, 11 patients showed a 9-point or greater increase in response to enzyme supplementation therapy, while one patient did not reach our threshold of a 9-point increase. Since the interobserver variation of $\mathrm{BMB}$ score was low, the precision was within approximately 1 point. Therefore $2.8 \times 1$ makes 3 points the required increase for detecting response with $95 \%$ confidence. The only patient that did not reach our fat fraction criteria was also nonresponsive to treatment according to $\mathrm{BMB}$ criteria. Only four of the 11 patients who satisfied the fat fraction criteria did not satisfy our BMB score criteria. In other words, there was similarity between fat fraction and BMB score outcomes in eight patients. When the responses of the two BMB components were evaluated separately, similar outcomes were obtained between the lumbar spine BMB scores and fat fractions in seven patients, while dissimilar outcomes were obtained in five patients. Similar outcomes were also obtained between femur BMB scores and fat fractions in four patients, while dissimilar outcomes were obtained in eight patients.

\section{DISCUSSION}

To our knowledge, Dixon QCSI is considered to be the only technique that can quantify axial bone marrow. Therefore it is thought to be the most desirable method for quantifying bone marrow invasion in Gaucher disease $(15,16,19-21)$. However, this technique is not widely available. In the determination of the amount of bone marrow involvement in Gaucher disease, the need for a semiquantitative scoring system that makes use of widely available conventional MR imaging is obvious, as already indicated in several articles $(11,21,26-28)$. All previously described scoring systems, to our knowledge, have been focused on only the peripheral bone marrow. The more dynamic axial bone marrow, which is thought to be the first site of invasion in Gaucher disease, has not been included in these scoring systems. This is in contrast to the literature concerning hematologic malignancies, in which analysis of bone marrow disease is focused on axial bone marrow $(8,12-14,23,24,32)$. Furthermore, it is known that skeletal complications occur most frequently in the lower extremities $(2,4,9,14,21,26)$. These changes, which include infarction, osteonecrosis, and fractures, are almost always irreversible (28). Therefore, a scoring system that includes these irreversible com- 
TABLE 4

Follow-up Results in 12 Patients

\begin{tabular}{|c|c|c|c|c|c|c|c|c|}
\hline \multirow{2}{*}{$\begin{array}{c}\text { Patient } \\
\text { No. }\end{array}$} & \multicolumn{2}{|c|}{ Fat Fraction } & \multicolumn{2}{|c|}{ Total BMB Score } & \multicolumn{2}{|c|}{ Lumbar Spine BMB Score } & \multicolumn{2}{|c|}{ Femur BMB Score } \\
\hline & Initial & Follow-up & Initial & Follow-up & Initial & Follow-up & Initial & Follow-up \\
\hline 1 & 0.11 & 0.28 & 13 & 10 & 6 & 3 & 7 & 7 \\
\hline 2 & 0.34 & 0.47 & 12 & 8 & 4 & 1 & 8 & 7 \\
\hline 3 & 0.11 & 0.38 & 12 & 7 & 5 & 0 & 7 & 7 \\
\hline 4 & 0.10 & 0.19 & 13 & 13 & 6 & 6 & 7 & 7 \\
\hline 5 & 0.22 & 0.41 & 9 & 4 & 5 & 1 & 4 & 3 \\
\hline 6 & 0.10 & 0.18 & 9 & 11 & 4 & 4 & 5 & 7 \\
\hline 7 & 0.23 & 0.42 & 10 & 1 & 5 & 1 & 5 & 0 \\
\hline 8 & 0.29 & 0.45 & 4 & 1 & 1 & 1 & 3 & 0 \\
\hline 9 & 0.18 & 0.47 & 8 & 1 & 5 & 1 & 3 & 0 \\
\hline 10 & 0.08 & 0.28 & 11 & 10 & 6 & 7 & 5 & 3 \\
\hline 11 & 0.18 & 0.35 & 9 & 10 & 5 & 6 & 4 & 4 \\
\hline 12 & 0.22 & 0.48 & 7 & 5 & 4 & 4 & 3 & 1 \\
\hline
\end{tabular}

Note.-Initial evaluation performed prior to treatment; follow-up evaluation performed at 40 months (mean) from initial evaluation. A higher score signifies more severe bone marrow involvement.

ponents would be relatively insensitive to subtle changes in bone marrow invasion such as those seen in Gaucher disease.

We believe ours is the first study that correlates Dixon QCSI as a bone marrow disease parameter with a semiquantitative scoring system based on both the change in signal intensity and the sites of involvement in axial and peripheral bone marrow. The introduced BMB scoring system shows a very good correlation with Dixon QCSI in untreated patients. The BMB score is a feasible evaluation method that has good interobserver and intraobserver variability when used by radiologists who are experienced in the analysis of MR images but are not experienced in the evaluation of Gaucher disease.

A good parameter of bone marrow invasion should also be able to demonstrate response to treatment. This has been described as an important quality of Dixon QCSI $(9,20)$. Dixon QCSI demonstrates response to therapy after 12 months (36). In our group, response to treatment was detected with Dixon QCSI in $11(92 \%)$ of 12 patients. Our results showed that BMB scoring could demonstrate response in nine patients (75\%). In other words, it seems that BMB is less sensitive than Dixon QCSI. By using our preset criteria, there was agreement between fat fraction and $\mathrm{BMB}$ score in eight of 12 patients. However, the studied population was small.

The scoring systems of the peripheral skeleton previously described by Terk et al (27) and Poll et al (28) also depicted response to therapy; the response rates in these studies were $67 \%$ and $63 \%$, respectively. The responses in both of these studies were scored in a binominal fashion (responder vs nonresponder). When we analyzed our data in 12 patients this manner, we found seven responders to treatment in the femur BMB group (58\%) and nine responders in the overall $\mathrm{BMB}$ group (75\%). In our population we found more responders when a combination of visual scoring of $\mathrm{BMB}$ of lumbar spine and femora was used. The femur BMB nonresponders in our population had irreversible changes like marrow infarction or avascular necrosis in the lower extremities. However, in some of them, response to treatment was demonstrated in the axial bone marrow. Therefore, one must consider that when no response is seen in the peripheral bone marrow, response may be present in the axial marrow. The drawback of our study was the limited number of patients in each group.

We conclude that the introduced BMB score has good correlation with the fat fraction measured with Dixon QCSI. The interobserver and intraobserver correlation make it reliable and easy to use for radiologists who are experienced in MR imaging but who do not have specific knowledge of Gaucher disease. It also seems to be able to demonstrate response to enzyme replacement therapy, although it is less sensitive in this than is Dixon QCSI. Further research should be performed to determine more definitely the strength of BMB as a follow-up bone marrow response parameter in Gaucher disease.

Acknowledgments: The authors thank Rezan Demir, MD, PhD, and Mirjam R. W. Evers-van Bavel for their aid in the preparation of this article. M. G. Dijkgraaf, PhD, is gratefully acknowledged for his support in the statistical analysis of the data.
References

1. Lee RE. The pathology of Gaucher disease. In: Desnick RJ, Gatt S, Grabowski GA, eds. Gaucher disease: a century of delineation and research. New York, NY: Liss, 1982; 177-217.

2. Beutler E, Grabowski G. Gaucher disease. In: Scriver CR, Sly WS, Valle D, eds. The metabolic basis of inherited disease. New York, NY: McGraw-Hill, 1995; 2641-2670.

3. Charrow J, Esplin JA, Gribble TJ, et al. Gaucher disease: recommendations on diagnosis, evaluation, and monitoring. Arch Intern Med 1998; 158:1754-1760.

4. Stowens DW, Teitelbaum SL, Kahn AJ, Barranger JA. Skeletal complications of Gaucher disease. Medicine 1985; 64:310_ 322 .

5. Barton NW, Brady RO, Dambrosia JM, et al. Dose dependent responses to macrophage-targeted glucocerebrosidase in a child with Gaucher disease. J Pediatr 1992; 120:277-280.

6. Hollak CE, Aerts JM, Goudsmit R, et al. Individualised low-dose alglucerase therapy for type 1 Gaucher's disease. Lancet 1995; 345:1474-1478.

7. Rosenthal DI, Doppelt SH, Mankin HJ, et al. Enzyme replacement therapy for Gaucher disease: skeletal responses to macrophage-targeted glucocerebrosidase. Pediatrics 1995; 96:629-637.

8. Vogler JB III, Murphy WA. Bone marrow imaging. Radiology 1988; 168:679-693.

9. Cremin BJ, Davey H, Goldblatt J. Skeletal complications of type 1 Gaucher disease: the magnetic resonance features. Clin Radiol 1990; 41:244-247.

10. Rosenthal DI, Scott JA, Barranger J, et al. Evaluation of Gaucher disease using magnetic resonance imaging. J Bone Joint Surg Am 1986; 68:802-808.

11. Hermann G, Shapiro RS, Abdelwahab IF, Grabowski G. MR imaging in adults with Gaucher disease type I: evaluation of marrow involvement and disease activity. Skeletal Radiol 1993; 22:247-251.

12. Lecouvet FE, Vande Berg BC, Michaux L et al. Stage III multiple myeloma: clinical and prognostic value of spinal bone marrow MR imaging. Radiology 1998; 209: $653-660$. 
13. Moulopoulos LA, Dimopoulos MA. Magnetic resonance imaging of the bone marrow in hematologic malignancies. Blood 1997; 90:2127-2147.

14. Steiner RM, Mitchell DG, Rao VM, Schweitzer ME. Magnetic resonance imaging of diffuse bone marrow disease. Radiol Clin N Am 1993; 31:383-409.

15. Rosenthal DI, Barton NW, McKusick KA, et al. Quantitative imaging of Gaucher disease. Radiology 1992; 185:841-845.

16. Johnson LA, Hoppel BE, Gerard EL, et al. Quantitative chemical shift imaging of vertebral bone marrow in patients with Gaucher disease. Radiology 1992; 182: 451-455.

17. Dixon WT. Simple proton spectroscopic imaging. Radiology 1984; 153:189-194.

18. Miller SPF, Zirzow GC, Doppelt SH, Brady RO, Barton NW. Analysis of the lipids of normal and Gaucher bone marrow. J Lab Clin Med 1996; 127:353-358

19. Maas M, Akkerman EM, Hollak CEM, Rademakers RP. Evaluation of skeletal involvement in M. Gaucher: use of quantitative chemical shift imaging (QCSI). Eur Radiol 1997; 7(suppl):206.

20. Rosenthal DI, Doppelt SH, Mankin HJ, et al. Enzyme replacement therapy for Gaucher disease: skeletal responses to macrophage-targeted glucocerebrosidase. Pediatrics 1995; 96:629-637.

21. O'Keeffe D, Rosenthal DI. Computed tomography and magnetic resonance imaging in Gaucher's disease. In: Bloem JL, Sartoris DJ, eds. MRI and CT of the musculoskeletal system: a text-atlas. Baltimore, Md: Williams \& Wilkins, 1992; 130-138.

22. Maas M, Akkerman EM, Venema HW, Stoker J, Den Heeten GJ. Dixon quantitative chemical shift MRI for bone marrow eval- uation in the lumbar spine: a reproducibility study in healthy volunteers. J Comput Assist Tomogr 2001; 25:691697.

23. Rosen BR, Fleming DM, Kushner DC, et al. Hematologic bone marrow disorders: quantitative chemical shift MR imaging. Radiology 1988; 169:799-804.

24. Gerard EL, Ferry JA, Amrein PC, et al. Compositional changes in vertebral bone marrow during treatment for acute leukemia: assessment with quantitative chemical shift imaging. Radiology 1992; 183: $39-46$

25. Gückel F, Brix G, Semmler W, et al. Proton chemical shift imaging of bone marrow for monitoring therapy in leukemia. J Comput Assist Tomogr 1990; 14:945959.

26. Terk MR, Esplin J, Lee K, Magre G, Colletti PM. MR imaging of patients with type 1 Gaucher's disease: relationship between bone and visceral changes. AJR Am J Roentgenol 1995; 165:599-604.

27. Terk MR, Dardashti S, Liebman HA. Bone marrow response in treated patients with Gaucher disease: evaluation by T1weighted magnetic resonance images and correlation with reduction in liver and spleen volume. Skeletal Radiol 2000; 29: 563-571.

28. Poll LW, Koch JA, vom Dahl S, et al. Magnetic resonance imaging of bone marrow changes in Gaucher disease during enzyme replacement therapy: first German long-term results. Skeletal Radiol 2001; 30:496-503

29. Sibille A, Eng CM, Kim SJ, Pastores G, Grabowski GA. Phenotype/genotype correlations in Gaucher disease type 1: clinical and therapeutic implications. Am J Hum Genet 1993; 52:1094-1101.
30. Sidransky E, Ginns EI. Phenotypic and genotypic heterogeneity in Gaucher disease: implications for genetic counseling. J Genet Counsel 1994; 3:13-22.

31. Boot RG, Hollak CEM, Verhoek M, et al. Glucocerebrosidase genotype of Gaucher patients in the Netherlands: limitations in prognostic value. Hum Mutat 1997; 10:348-358.

32. Moulopoulos LA, Varma DGK, Dimopoulos MA, et al. Multiple myeloma: spinal MR imaging in patients with untreated newly diagnosed disease. Radiology 1992; 185:833-840.

33. Algra PR, Bloem JL, Valk J. Disappearance of the basivertebral vein: a new MR imaging sign of bone marrow disease. AJR Am J Roentgenol 1991; 157:1129-1130.

34. Cummings SR, Black DB. Should perimenopausal women be screened for osteoporosis? Ann Intern Med 1986; 104: 817-823.

35. Akkerman EM, Maas M. A region-growing algorithm to simultaneously remove dephasing influences and separate fat and water in two-point Dixon imaging (abstr). In: Proceedings of the Society of Magnetic Resonance in Medicine and the European Society of Magnetic Resonance in Medicine and Biology. Berkeley, Calif: Society of Magnetic Resonance in Medicine, 1995; 649 .

36. Hollak C, Maas M, Akkerman EM, den Heeten A, Aerts H. Dixon quantitative chemical shift imaging is a sensitive tool for the evaluation of bone marrow responses to individualized doses of enzyme supplementation therapy in type 1 Gaucher disesase. Blood Cells Mol Dis 2001; 27:1005-1012. 